\title{
Erratum to: HVOF Colloquium 2015: High Velocity Oxy-Fuel Flame Spraying in Focus
}

\section{Erratum to: Journal of Thermal Spray Technology 4:590-591 \\ DOI: 10.1007/s11666-015-0230-6}

We regret that the title of the original article was incorrect. The correct title should be "HVOF Colloquium 2015: High Velocity Oxy-Fuel Flame Spraying in Focus." as shown in this erratum. 